\title{
Cusa-Huygens, Wilker and Huygens Type Inequalities for Generalized Hyperbolic Functions
}

\author{
Kwara Nantomah
}

Department of Mathematics, Faculty of Mathematical Sciences,

C.K. Tedam University of Technology and Applied Sciences,

P. O. Box 24, Navrongo, Upper-East Region, Ghana

e-mail: knantomah@cktutas.edu.gh

\begin{abstract}
In this paper, we establish Cusa-Huygens, Wilker and Huygens type inequalities for certain generalizations of the hyperbolic functions. From the established results, we recover some previous results as particular cases.
\end{abstract}

\section{Introduction}

The inequality

$$
\frac{\sin z}{z}<\frac{\cos z+2}{3}, \quad 0<z<\frac{\pi}{2},
$$

is known in the literature as Cusa-Huygens inequality. Its hyperbolic counterpart, which is given as

$$
\frac{\sinh z}{z}<\frac{\cosh z+2}{3}, \quad x>0,
$$

was established by Neuman and Sandor [14]. The inequality

$$
\left(\frac{\sin z}{z}\right)^{2}+\frac{\tan z}{z}>2, \quad 0<z<\frac{\pi}{2}
$$

which is known as Wilker inequality was first proposed in the classic work 21, p.55] and subsequently attrated the attention of other researchers. In [23, $\mathrm{Wu}$

Received: September 3, 2020; Accepted: September 29, 2020

2010 Mathematics Subject Classification: 33B10, 33Bxx, 26D05.

Keywords and phrases: generalized hyperbolic functions, Cusa-Huygens inequality, Wilker inequality, Huygens inequality. 
and Srivastava proved the Wilker-type inequality

$$
\left(\frac{z}{\sin z}\right)^{2}+\frac{z}{\tan z}>2, \quad 0<z<\frac{\pi}{2} \text {. }
$$

The hyperbolic counterpart of (3) was established by $\mathrm{Zu}$ [24] as

$$
\left(\frac{\sinh z}{z}\right)^{2}+\frac{\tanh z}{z}>2, \quad z \in \mathbb{R} \backslash\{0\}
$$

Also, the hyperbolic counterpart of (4) was established by Wu and Debnath [22] as

$$
\left(\frac{z}{\sinh z}\right)^{2}+\frac{z}{\tanh z}>2, \quad z \in \mathbb{R} \backslash\{0\} .
$$

Another inequality of interest is the Huygens inequality which is given as [17]

$$
2 \frac{\sin z}{z}+\frac{\tan z}{z}>3, \quad 0<z<\frac{\pi}{2}
$$

and its hyperbolic counterpart given as [14]

$$
2 \frac{\sinh z}{z}+\frac{\tanh z}{z}>3, \quad z \in \mathbb{R} \backslash\{0\} .
$$

Due to their usefulness, these elegant inequalities have been studied extensively and in diverse ways by several researchers. See for example [2], [3], [4], [5], [6], [7], [8], [12], [13], [15], [16], [18, [19], [20], [22], 24], 25], [26] and the related references therein.

Also, in a recent work, the Huygens-type inequality

$$
\frac{2}{\cosh z}+\cosh z>\frac{\sinh z}{z}+2 \frac{\tanh z}{z}>\frac{1}{\cosh z}+2, \quad z \in \mathbb{R} \backslash\{0\},
$$

was established among other things by Bagul and Chesneau [1].

Motivated by the results (2), (5), (6), (8) and (9), the objective of this paper is to establish analogous inequalities concerning certain generalizations of the hyperbolic functions. The established results serve as generalizations of the previous results. 


\section{Preliminary Definitions}

In a bid to generalize a previous work [9], the authors of [10] gave the following generalizations of the hyperbolic functions.

Definition 2.1. The generalized hyperbolic cosine, hyperbolic sine and hyperbolic tangent functions are respectively defined as [10]

$$
\begin{gathered}
\cosh _{a}(z)=\frac{a^{z}+a^{-z}}{2}, \\
\sinh _{a}(z)=\frac{a^{z}-a^{-z}}{2}, \\
\tanh _{a}(z)=\frac{\sinh _{a}(z)}{\cosh _{a}(z)}=\frac{a^{z}-a^{-z}}{a^{z}+a^{-z}}=1-\frac{2}{1+a^{2 z}},
\end{gathered}
$$

where $a>1$ and $z \in \mathbb{R}$.

These generalized functions satisfy the following identities.

$$
\begin{gathered}
\cosh _{a}(z)+\sinh _{a}(z)=a^{z}, \\
\cosh _{a}(z)-\sinh _{a}(z)=a^{-z}, \\
\left(\cosh _{a}(z)\right)^{\prime}=(\ln a) \sinh _{a}(z), \\
\left(\sinh _{a}(z)\right)^{\prime}=(\ln a) \cosh _{a}(z), \\
\left(\tanh _{a}(z)\right)^{\prime}=\frac{\ln a}{\cosh _{a}^{2}(z)}, \\
\left(\cosh _{a}(z)\right)^{\prime \prime}+\left(\sinh _{a}(z)\right)^{\prime \prime}=(\ln a)^{2} a^{z}, \\
\left(\cosh _{a}(z)\right)^{\prime \prime}-\left(\sinh _{a}(z)\right)^{\prime \prime}=(\ln a)^{2} a^{-z}, \\
\cosh _{a}^{2}(z)+\sinh _{a}^{2}(z)=\cosh _{a}(2 z), \\
\cosh _{a}^{2}(z)-\sinh _{a}^{2}(z)=1, \\
2 \sinh _{a}(z) \cosh _{a}(z)=\sinh _{a}(2 z), \\
\cosh _{a}^{2}(z)=\frac{\cosh _{a}(2 z)+1}{2},
\end{gathered}
$$




$$
\sinh _{a}^{2}(z)=\frac{\cosh _{a}(2 z)-1}{2} .
$$

The generalized hyperbolic secant, hyperbolic cosecant and hyperbolic cotangent functions are respectively defined as

$$
\operatorname{sech}_{a}(z)=\frac{1}{\cosh _{a}(z)}, \quad \operatorname{cosech}_{a}(z)=\frac{1}{\sinh _{a}(z)}, \quad \operatorname{coth}_{a}(z)=\frac{1}{\tanh _{a}(z)} .
$$

As pointed out in [10], several other identities can be derived from (10), (11) and (12). When $a=e$, where $e=2.71828 \ldots$ is the Euler's number, then the above definitions and identities reduce to their ordinary counterparts.

\section{Results and Discussion}

Lemma 3.1. The inequality

$$
\cosh _{a}(z)<\left(\frac{\sinh _{a}(z)}{z}\right)^{3}
$$

holds for $z \in \mathbb{R} \backslash\{0\}$.

Proof. Inequality (26) has been proved in [11] for $z>0$. Now let $z<0$ so that $-z>0$. Then

$$
\cosh _{a}(z)=\cosh _{a}(-z)<\left(\frac{\sinh _{a}(-z)}{-z}\right)^{3}=\left(\frac{\sinh _{a}(z)}{z}\right)^{3},
$$

which completes the proof.

Since the function $\frac{\sinh _{a}(z)}{z}$ is increasing for $z>0$ and decreasing for $z<0$, then Lemma 3.1 implies the following generalized result.

Lemma 3.2. The inequality

$$
\cosh _{a}(z)<\left(\frac{\sinh _{a}(z)}{z}\right)^{v}
$$

holds for $z \in \mathbb{R} \backslash\{0\}$ and $v \geq 3$. 
Lemma 3.3 ([11]). For $z \in \mathbb{R} \backslash\{0\}$, the inequality

$$
\frac{\ln a}{\cosh _{a}(z)}<\frac{\sinh _{a}(z)}{z}<(\ln a) \cosh _{a}(z),
$$

holds.

Lemma 3.4 (Young's Inequality). Let $x, y \geq 0, r, s \in(0,1)$ such that $r+s=1$. Then,

$$
x^{r} y^{s} \leq r x+s y .
$$

Theorem 3.5. The inequality

$$
\frac{\sinh _{a}(z)}{z}<\frac{2 \ln a+(\ln a) \cosh _{a}(z)}{3}
$$

holds for $z \in \mathbb{R} \backslash\{0\}$.

Proof. Since the functions in each term of the inequality are even, it suffices to prove the case for $z>0$. Let $z>0$ and $h$ be defined as

$$
h(z)=\frac{2 z+z \cosh _{a}(z)}{\sinh _{a}(z)} .
$$

Then

$$
\begin{aligned}
h^{\prime}(z) & =\frac{1}{\sinh _{a}^{2}(z)}\left[2 \sinh _{a}(z)+\cosh _{a}(z) \sinh _{a}(z)-2 z(\ln a) \cosh _{a}(z)-(\ln a) z\right] \\
& =\frac{1}{\sinh _{a}^{2}(z)} \phi(z)
\end{aligned}
$$

and then

$$
\begin{aligned}
\phi^{\prime}(z) & =(\ln a)\left[\cosh _{a}(2 z)-2(\ln a) z \sinh _{a}(z)-1\right] \\
& =2(\ln a) \sinh _{a}(z)\left[\sinh _{a}(z)-(\ln a) z\right]>0,
\end{aligned}
$$

since $\frac{\sinh _{a}(z)}{z}>\ln a$ for $z \in(0, \infty)$. Hence $\phi(z)$ is increasing and consequently, $\phi(z)>\phi(0)=0$. Thus $h(z)$ is increasing. Hence

$$
h(z)>\lim _{z \rightarrow 0} h(z)=\frac{3}{\ln a},
$$

which gives 30 . 
Remark 3.6. When $a=e$, then inequality 30 reduces to the hyperbolic Cusa-Huygens inequality (2).

Theorem 3.7. The inequalities

$$
\begin{gathered}
\left(\frac{\sinh _{a}(z)}{z}\right)^{2}+\frac{\tanh _{a}(z)}{z}>2, \\
\left(\frac{z}{\sinh _{a}(z)}\right)^{2}+\frac{z}{\tanh _{a}(z)}>\frac{1+\ln a}{(\ln a)^{2}}, \quad 1<a \leq e,
\end{gathered}
$$

hold for $z \in \mathbb{R} \backslash\{0\}$.

Proof. Let $z \in \mathbb{R} \backslash\{0\}$. Then by the AM-GM inequality and Lemma 3.1, we obtain

$$
\begin{aligned}
\left(\frac{\sinh _{a}(z)}{z}\right)^{2}+\frac{\tanh _{a}(z)}{z} & \geq 2 \sqrt{\left(\frac{\sinh _{a}(z)}{z}\right)^{2} \frac{\tanh _{a}(z)}{z}} \\
& =2 \sqrt{\left(\frac{\sinh _{a}(z)}{z}\right)^{3} \frac{1}{\cosh _{a}(z)}} \\
& >2,
\end{aligned}
$$

which gives (31). To prove (32), it suffices to prove the case for $z>0$. Let $z>0$ and define $\psi(z)$ by

$$
\psi(z)=\left(\frac{z}{\sinh _{a}(z)}\right)^{2}+\frac{z}{\tanh _{a}(z)},
$$

where $1<a \leq e$. Then by differentiating, applying the AM-GM inequality and Lemma 3.1, we obtain

$$
\begin{aligned}
& \psi^{\prime}(z) \\
& =\frac{1}{\sinh _{a}^{3}(z)}\left[\sinh _{a}^{2}(z) \cosh _{a}(z)+(2-\ln a) z \sinh _{a}(z)-2(\ln a) z^{2} \cosh _{a}(z)\right] \\
& \geq \frac{1}{\sinh _{a}^{3}(z)}\left[2 \sqrt{\sinh _{a}^{2}(z) \cosh _{a}(z) \cdot(2-\ln a) z \sinh _{a}(z)}-2(\ln a) z^{2} \cosh _{a}(z)\right] \\
& =\frac{2 z^{2}}{\sinh _{a}^{3}(z)} \cdot \sqrt{\cosh _{a}(z)}\left[\sqrt{2-\ln a} \sqrt{\left(\frac{\sinh _{a}(z)}{z}\right)^{3}}-(\ln a) \sqrt{\cosh _{a}(z)}\right] \\
& >0
\end{aligned}
$$


Thus $\psi(z)$ is increasing. Hence

$$
\psi(z)>\lim _{z \rightarrow 0} \psi(z)=\frac{1+\ln a}{(\ln a)^{2}},
$$

which gives 32 .

Remark 3.8. When $a=e$, then the inequalities (31) and (31) reduce to the hyperbolic Wilker-type inequalities (5) and (6) respectively.

Theorem 3.9. The inequality

$$
\left(\frac{\sinh _{a}(z)}{z}\right)^{2}+\frac{\tanh _{a}(z)}{z}>\left(\frac{z}{\sinh _{a}(z)}\right)^{2}+\frac{z}{\tanh _{a}(z)}
$$

holds for $z \in \mathbb{R} \backslash\{0\}$.

Proof. Using the fact that $\left(A^{2}+B\right) /\left(1 / A^{2}+1 / B\right)=A^{2} B$, together with Lemma 3.1. we obtain

$$
\begin{aligned}
\frac{\left(\frac{\sinh _{a}(z)}{z}\right)^{2}+\frac{\tanh _{a}(z)}{z}}{\left(\frac{z}{\sinh _{a}(z)}\right)^{2}+\frac{z}{\tanh _{a}(z)}} & =\left(\frac{\sinh _{a}(z)}{z}\right)^{2} \frac{\tanh _{a}(z)}{z} \\
& =\left(\frac{\sinh _{a}(z)}{z}\right)^{3} \frac{1}{\cosh _{a}(z)} \\
& >1,
\end{aligned}
$$

which concludes the proof.

Theorem 3.10. Let $\alpha, \beta \in(0,1)$ such that $\alpha+\beta=1$. Then the inequality

$$
\alpha\left(\frac{\sinh _{a}(z)}{z}\right)^{2}+\beta\left(\frac{\tanh _{a}(z)}{z}\right)>(\ln a)^{2(\alpha-\beta)},
$$

holds for $z \in \mathbb{R} \backslash\{0\}$. 
Proof. Let $z \in \mathbb{R} \backslash\{0\}$. Then Youngs inequality 29 and Lemma 3.1 imply that

$$
\begin{aligned}
\alpha\left(\frac{\sinh _{a}(z)}{z}\right)^{2}+\beta\left(\frac{\tanh _{a}(z)}{z}\right) & \geq\left(\frac{\sinh _{a}(z)}{z}\right)^{2 \alpha}\left(\frac{\tanh _{a}(z)}{z}\right)^{\beta} \\
& =\left(\frac{\sinh _{a}(z)}{z}\right)^{2 \alpha+\beta}\left(\frac{1}{\cosh _{a}(z)}\right)^{\beta} \\
& >\left(\frac{\sinh _{a}(z)}{z}\right)^{2 \alpha+\beta}\left(\frac{\sinh _{a}(z)}{z}\right)^{-3 \beta} \\
& =\left(\frac{\sinh _{a}(z)}{z}\right)^{2(\alpha-\beta)} \\
& >(\ln a)^{2(\alpha-\beta)}
\end{aligned}
$$

which completes the proof.

Remark 3.11. If $\alpha=\beta=\frac{1}{2}$, then (34) reduces to 31.

Theorem 3.12. The inequality

$$
2 \frac{\sinh _{a}(z)}{z}+\frac{\tanh _{a}(z)}{z}>3 \ln a
$$

holds for $z \in \mathbb{R} \backslash\{0\}$.

Proof. It suffices to prove the case for $z>0$. Let $z>0$ and let $h$ be defined as

$$
h(z)=2 \frac{\sinh _{a}(z)}{z}+\frac{\tanh _{a}(z)}{z} .
$$

Then

$$
\begin{aligned}
z^{2} h^{\prime}(z) & =2(\ln a) z \cosh _{a}(z)-2 \sinh _{a}(z)+(\ln a) z \operatorname{sech}_{a}^{2}(z)-\tanh _{a}(z) \\
& =\theta(z)
\end{aligned}
$$

and

$$
\begin{aligned}
\theta^{\prime}(z) & =2(\ln a)^{2} z \sinh _{a}(z)-2(\ln a)^{2} z \tanh _{a}(z) \operatorname{sech}_{a}^{2}(z) \\
& =2(\ln a)^{2} z \sinh _{a}(z)\left[1-\frac{1}{\cosh _{a}^{3}(z)}\right]>0
\end{aligned}
$$


which shows that $\theta(z)$ is increasing. Hence $\theta(z)>\theta(0)=0$. Thus, $h(z)$ is increasing and consequently,

$$
h(z)>\lim _{z \rightarrow 0} h(z)=3 \ln a
$$

which yields (35).

Remark 3.13. When $a=e$, then inequality 35 reduces to 8 .

Theorem 3.14. The inequality

$$
2 \frac{z}{\sinh _{a}(z)}+\frac{z}{\tanh _{a}(z)}>\frac{3}{\ln a},
$$

holds for $z \in \mathbb{R} \backslash\{0\}$.

Proof. It suffices to prove the case for $z>0$. Let $z>0$ and $\delta$ be defined as

$$
\delta(z)=2 \frac{z}{\sinh _{a}(z)}+\frac{z}{\tanh _{a}(z)} .
$$

Then

$$
\delta^{\prime}(z)=\frac{1}{\sinh _{a}^{2}(z)}\left[2 \sinh _{a}(z)-z \ln a+\cosh _{a}(z)\left(\sinh _{a}(z)-z \ln a\right)\right]>0
$$

since $\sinh _{a}(z)>z \ln a$. Hence $\delta(z)$ is increasing and consequently,

$$
\delta(z)>\lim _{z \rightarrow 0} \delta(z)=\frac{3}{\ln a}
$$

which yields 36 .

Theorem 3.15. The inequality

$$
(m+1) \frac{\sinh _{a}(z)}{z}+m \frac{\tanh _{a}(z)}{z}>m \frac{\sinh _{a}(z)}{z}+(m+1) \frac{\tanh _{a}(z)}{z},
$$

holds for $z \in \mathbb{R} \backslash\{0\}$ and $m \in \mathbb{N} \cup\{0\}$. 
Proof. $z \in \mathbb{R} \backslash\{0\}$. Then

$$
\frac{\sinh _{a}(z)}{z}-\frac{\tanh _{a}(z)}{z}=\frac{\sinh _{a}(z)}{z}\left[1-\frac{1}{\cosh _{a}(z)}\right]>0,
$$

since $\cosh _{a}(z)>1$ for $z \in \mathbb{R}$. That is

$$
\frac{\sinh _{a}(z)}{z}>\frac{\tanh _{a}(z)}{z} .
$$

Adding $m\left(\frac{\sinh _{a}(z)}{z}+\frac{\tanh _{a}(z)}{z}\right)$ to both sides of $(38)$ completes the proof.

Theorem 3.16. The inequality

$$
\frac{2 \ln a}{\cosh _{a}(z)}+(\ln a) \cosh _{a}(z)>\frac{\sinh _{a}(z)}{z}+2 \frac{\tanh _{a}(z)}{z}>\frac{\ln a}{\cosh _{a}(z)}+2 \ln a,
$$

holds for $z \in \mathbb{R} \backslash\{0\}$.

Proof. It suffices to prove the case for $z>0$. Let $z>0$ and $f$ be defined as

$$
f(z)=2(\ln a) z+(\ln a) z \cosh _{a}^{2}(z)-\sinh _{a}(z) \cosh _{a}(z)-2 \sinh _{a}(z) .
$$

Then

$$
f^{\prime}(z)=2(\ln a)+2(\ln a)^{2} z \cosh _{a}(z) \sinh _{a}(z)-(\ln a) \sinh _{a}^{2}(z)-2(\ln a) \cosh _{a}(z),
$$

and then

$$
f^{\prime \prime}(z)=2(\ln a)^{3} z \sinh _{a}^{2}(z)+2(\ln a)^{2}\left[(\ln a) z \cosh _{a}^{2}(z)-\sinh _{a}(z)\right]>0,
$$

since $\sinh _{a}(z)<(\ln a) z \cosh _{a}(z)$ (see Lemma 3.3) and $\cosh _{a}(z)<\cosh _{a}^{2}(z)$. Hence $f^{\prime}(z)$ is increasing and so, $f^{\prime}(z)>f^{\prime}(0)=0$. Thus, $f(z)$ is increasing and so $f(z)>f(0)=0$. This yields the left-hand side of $(39)$. Next, for $z>0$, let $g$ be defined as

$$
g(z)=\sinh _{a}(z) \cosh _{a}(z)+2 \sinh _{a}(z)-(\ln a) z-2(\ln a) z \cosh _{a}(z) .
$$

Then

$$
g^{\prime}(z)=(\ln a) \cosh _{a}^{2}(z)+(\ln a) \sinh _{a}^{2}(z)-\ln a-2(\ln a)^{2} z \sinh _{a}(z)
$$


and then

$$
\begin{aligned}
g^{\prime \prime}(z) & =2(\ln a)^{2} \cosh _{a}(z)\left[\sinh _{a}(z)-(\ln a) z\right]+2(\ln a)^{2} \sinh _{a}(z)\left[\cosh _{a}(z)-1\right] \\
& >0
\end{aligned}
$$

since $\sinh _{a}(z)>(\ln a) z$ and $\cosh _{a}(z)>1$. Hence $g^{\prime}(z)$ is increasing and so, $g^{\prime}(z)>g^{\prime}(0)=0$. Thus, $g(z)$ is increasing and so $g(z)>g(0)=0$. This yields the right-hand side of $(39)$ and that completes the proof.

Remark 3.17. When $a=e$, then inequality (39) reduces to (9).

Remark 3.18. By (37) and (39), we have

$$
2 \frac{\sinh _{a}(z)}{z}+\frac{\tanh _{a}(z)}{z}>\frac{\ln a}{\cosh _{a}(z)}+2 \ln a .
$$

This is however weaker than inequality (35).

\section{Acknowledgement}

The author is grateful to the anonymous referees for thorough reading of the manuscript.

\section{References}

[1] Y.J. Bagul and C. Chesneau, Two double sided inequalities involving sinc and hyperbolic sinc functions, Int. J. Open Problems Compt. Math. 12(4) (2019), 15-20.

[2] Y.J. Bagul and C. Chesneau, Some new simple inequalities involving exponential, trigonometric and hyperbolic functions, Cubo 21(1) (2019), 21-35. https://doi.org/10.4067/S0719-06462019000100021

[3] C.-P. Chen, Wilker and Huygens type inequalities for the Lemniscate functions, J. Math. Inequal. 6(4) (2012), 673-684. https://doi.org/10.7153/jmi-06-65

[4] C.-P. Chen and W.-S. Cheung, Sharp Cusa and Becker-Stark inequalities, J. Inequal. Appl. 2011, Article No. 136, 6 pp.

https://doi.org/10.1186/1029-242X-2011-136 
[5] C.-P. Chen and J. Sandor, Inequality chains for Wilker, Huygens and Lazarevic type inequalities, J. Math. Inequal. 8(1) (2018), 55-67. https://doi.org/10.7153/jmi-08-02

[6] L.-G. Huang, L. Yin, Y.-L. Wang and X.-L. Lin, Some Wilker and Cusa type inequalities for generalized trigonometric and hyperbolic functions, J. Inequal. Appl. 2018, Article No. 52, 8 pp. https://doi.org/10.1186/s13660-018-1644-8

[7] B. Malesevic, T. Lutovac, M. Rasajski and C. Mortici, Extensions of the natural approach to refinements and generalizations of some trigonometric inequalities, Adv. Difference Equ. 2018, Paper No. 90, 15 pp. https://doi.org/10.1186/s13662-018-1545-7

[8] C. Mortici, The natural approach of Wilker-Cusa-Huygens inequalities, Math. Inequal. Appl. 14 (2011), 535-541. https://doi.org/10.7153/mia-14-46

[9] K. Nantomah, On some properties of the sigmoid function, Asia Mathematika 3(1) (2019), 79-90.

[10] K. Nantomah, C. A. Okpoti and S. Nasiru, On a generalized sigmoid function and its properties, Asian J. Math. Appl. 2020, Article ID ama0527, 11 pp.

[11] K. Nantomah and E. Prempeh, Some inequalities for generalized hyperbolic functions, Moroccan J. Pure Appl. Anal. 6(1) (2020), 76-92. https://doi.org/10.2478/mjpaa-2020-0007

[12] E. Neuman, On Wilker and Huygens type inequalities, Math. Inequal. Appl. 15(2) (2012), 271-279. https://doi.org/10.7153/mia-15-22

[13] E. Neuman, Wilker and Huygens-type inequalities for the generalized trigonometric and for the generalized hyperbolic functions, Appl. Math. Comput. 230 (2014), 211-217. https://doi.org/10.1016/j.amc.2013.12.136

[14] E. Neuman and J. Sandor, On some inequalities involving trigonometric and hyperbolic functions with emphasis on the Cusa-Huygens, Wilker, and Huygens inequalities, Math. Inequal. Appl. 13(4) (2010), 715-723.

https://doi.org/10.7153/mia-13-50

[15] J. Sandor, On Huygens' inequalities and the theory of means, Int. J. Math. Math. Sci. 2012, Article ID 597490, 9 pp. https://doi.org/10.1155/2012/597490 
[16] J. Sandor, Sharp Cusa-Huygens and related inequalities, Notes on Number Theory and Discrete Mathematics 19(1) (2013), 50-54.

[17] J. Sandor and M. Bencze, On Huygens' trigonometric inequality, RGMIA Res. Rep. Coll. 8(3) (2005), Art. 14.

[18] J. Sandor and R. Olah-Gal, On Cusa-Huygens type trigonometric and hyperbolic inequalities, Acta Univ. Sapientiae Math. 4(2) (2012), 145-153.

[19] J.B. Wilker, J.S. Sumner, A.A. Jagers, Michael Vowe and Jean Anglesio, E3306, Amer. Math. Monthly 98(3) (1991), 264-267. https://doi.org/10.2307/2325035

[20] Z. Sun and L. Zhu, On new Wilker-type inequalities, ISRN Math. Anal. 2011, Article ID 681702,7 pp. https://doi.org/10.5402/2011/681702

[21] H.L. Montgomery, J.D. Vaaler, J. Delany, D.E. Knuth, D. Vialetto, M.S. Klamkin and J.B. Wilker, Elementary Problems: E3301-E3306, Amer. Math. Monthly 96(1) (1989), 54-55. https://doi.org/10.2307/2323259

[22] S. Wu and L. Debnath, Wilker-type inequalities for hyperbolic functions, Appl. Math. Lett. 25 (2012), 837-842.https://doi.org/10.1016/j.aml.2011.10.028

[23] S.-H. Wu and H.M. Srivastava, A weighted and exponential generalization of Wilker's inequality and its applications, Integral Transforms Spec. Funct. 18(7-8) (2007), 529-535. https://doi.org/10.1080/10652460701284164

[24] L. Zhu, On Wilker-type inequalities, Math. Inequal. Appl. 10(4) (2007), 727-731. https://doi.org/10.7153/mia-10-67

[25] L. Zhu, A source of inequalities for circular functions, Comput. Math. Appl. 58 (2009), 1998-2004. https://doi.org/10.1016/j.camwa.2009.07.076

[26] L. Zhu, New inequalities of Wilker's type for hyperbolic functions, AIMS Mathematics 5(1) (2020), 376-384. https://doi.org/10.3934/math.2020025

This is an open access article distributed under the terms of the Creative Commons Attribution License (http://creativecommons.org/licenses/by/4.0/), which permits unrestricted, use, distribution and reproduction in any medium, or format for any purpose, even commercially provided the work is properly cited. 